\title{
Generative Design of Stable Semiconductor Materials USING DEEP LEARNING AND DFT
}

\author{
Edirisuriya M. Dilanga Siriwardane \\ Department of Computer Science and Engineering \\ University of South Carolina \\ Columbia, SC 29201 \\ Department of Computer Science and Engineering \\ University of Moratuwa \\ Moratuwa, Sri Lanka \\ Indika Perera \\ Department of Computer Science and Engineering \\ University of Moratuwa \\ Moratuwa, Sri Lanka
}

\author{
Yong Zhao \\ Department of Computer Science and Engineering \\ University of South Carolina \\ Columbia, SC 29201
}

\begin{abstract}
Semiconductor device technology has exceptionally developed in complexity since discovering the bipolar transistor. With the rapid advancement of various technologies, semiconductors with distinct properties are essential. Recently, deep-learning, data-mining, and density functional theory (DFT)based high-throughput calculations were widely performed to discover potential semiconductors for diverse applications. CubicGAN is a generative adversarial network where high-throughput analyses were done to uncover mechanically and dynamically stable materials with the assistance of DFT. In our work, we screened the semiconductors using a binary classifier from materials found from the CubicGAN. Next, we performed DFT computations to study their thermodynamic stability based on energy-above-hull and formation energy. According to our studies, 12 stable semiconductors were found with a particular class of materials, which we label as $\mathrm{AA}^{\prime} \mathrm{MH}_{6}$. Those are $\mathrm{BaNaRhH}_{6}, \mathrm{BaSrZnH}_{6}, \mathrm{BaCsAlH}_{6}, \mathrm{SrTlIrH}_{6}, \mathrm{KNaNiH}_{6}, \mathrm{NaYRuH}_{6}, \mathrm{CsKSiH}_{6}, \mathrm{CaScMnH}_{6}$, $\mathrm{YZnMnH}_{6}, \mathrm{NaZrMnH}_{6}, \mathrm{AgZrMnH}_{6}, \mathrm{AgZrMnH}_{6}$, and $\mathrm{ScZnMnH}_{6}$. It could be shown that $\mathrm{AA}^{\prime} \mathrm{MH}_{6}$ with $\mathrm{M}=\mathrm{Mn}$ and $\mathrm{NaYRuH}_{6}$ semiconductors have considerably different structural, mechanical, and thermodynamic properties compared to the rest of the $\mathrm{AA}^{\prime} \mathrm{MH}_{6}$ semiconductors. In this study, The maximum bandgap found was approximately $3.3 \mathrm{eV}$ from $\mathrm{KNaNiH}_{6}$, while the minimum bandgap was about $1.3 \mathrm{eV}$ from $\mathrm{CaScMnH}_{6} . \mathrm{BaNaRhH}_{6}, \mathrm{BaCsAlH}_{6}, \mathrm{CsKSiH}_{6}, \mathrm{KNaNiH}_{6}$, and $\mathrm{NaYRuH}_{6}$ were identified as wide-bandgap semiconductors, where bandgaps are greater than $2 \mathrm{eV}$. Furthermore, $\mathrm{BaSrZnH}_{6}$ and $\mathrm{KNaNiH}_{6}$ are a direct bandgap semiconductors, whereas other $\mathrm{AA}^{\prime} \mathrm{MH}_{6}$ semiconductors exhibit indirect bandgaps.
\end{abstract}

Keywords Deep learning $\cdot$ Semiconductors $\cdot$ Density Functional Theory $\cdot$ Generative Adversarial Networks

\section{Introduction}

Semiconductors are essential components of modern devices that use transistors, light-emitting diodes [1], integrated circuits [2], photovoltaic [3], solar cells [4], and so on [5, 6, 7]. Semiconductors exhibit variable resistance since electron flow can be controlled by light and heat. Therefore, these materials can be used for energy conversion, and digital switching [8]. The elemental semiconductors found from Group XIV in the periodic table, like Si and Ge, and the compounds of Ge are widely used in electronics, photovoltaic and optoelectronic devices. However, semiconductors with various properties are required for industrial applications [8, 9]. For instance, excellent thermal conductivity 
and electric field breakdown strength, and also wide bandgap of $\mathrm{SiC}$ semiconductor make it a suitable material for high-temperature, high-power, high-frequency, and high-radiation conditions [10]. Thus, computational approaches for exploring novel semiconductors are essential to enhance future technologies. High-throughput screening with the aid of first-principles calculations was performed by several groups to discover novel optoelectronic semiconductors. Setyawan et al. and Ortiz et al. reported the high-throughput screening and data-mining frameworks to investigate bandgap materials for radiation detection [11, 12, 13]. High throughput material screening by Zhao et al. found that $\mathrm{Cu}$-In-based Halide Perovskite as potential photovoltaic solar absorbers [14, 13]. Based on 4507 hypothetical materials, Li et al. suggest 23 candidates for light-emitting applications, and 13 potential compounds for solar cell technologies [15, 13]. Such examples indicate that high-throughput screening can now be used to explore promising semiconductor materials.

Generative adversarial networks (GANs) are a kind of generative models that learn patterns/distribution from input data [16]. GANs use two sub-models to train a generative model. The generator model generates fake data, and the discriminator model learns to tell fake data from real data. The two sub-models are trained simultaneously to achieve a Nash Equilibrium: the generator can generate data that the discriminator can recognize half the chance. Wasserstein distance [17] and gradient penalty [18] are introduced during training in order to overcome mode collapse and improve the training stability in original GANs [16]. There are a limited number of works that leverage GANs to generate crystal structures in material science. The reasons behind that are: 1) Crystal structures have so many formations, such as a different number of elements and number of atoms in a unit cell. It is hard to come up with an unified representation to make GANs learn from them like images or text; 2) GANs used in computer vision cannot generate crystal structures that satisfy physics or symmetric constraints. For instance, GANs easily generate materials that are not recognizable or that have crowd atoms in a unit cell. CrystalGAN [19] is believed to be the first work that uses GANs to generate materials. It applies CyClyGAN [20] to simple systems mapping ternary a hydride into another. In [21], Kim et al. use WGAN-GP [18] to train a generative model to generate Mg-Mn-O systems with atom coordinates as the input. All the works above only consider a simple or specific family of materials at a limited scale. CubicGAN proposed by Zhao et al. [22], however, is the first work that generates materials at a large scale.

In this research, we developed a binary classifier to filter the semiconductors/Insulators (non-metals) from the dynamically stable quaternary Cubic materials discovered using the CubicGAN model, where high-throughput calculations were done with the assistance of a GAN model and density functional theory (DFT). We studied the most important elemental and electronic properties, which are helpful to distinguish the non-metals and metals using the machine learning models. In addition, we carried out DFT calculations for those semiconductors to corroborate the thermodynamic stability and semiconductor properties. As a result, we find that 12 cubic semiconductors of a particular class of materials, which we label as $\mathrm{AA}^{\prime} \mathrm{MH}_{6}$, are thermodynamically stable against their competing phases. We further performed the DFT calculations to study their structural, mechanical, thermodynamic, and electronic properties. Our results show that $\mathrm{AA}^{\prime} \mathrm{MnH}_{6}$ and $\mathrm{NaYRuH}_{6}$ have higher $\mathrm{C}_{i i}(\mathrm{i}=1,2,3)$ elastic constants, bulk modulus, shear modulus, and Young's modulus compared to the respective mechanical properties of the rest of the $\mathrm{AA}^{\prime} \mathrm{MH}_{6}$ materials. At temperatures less than $200 \mathrm{~K}, \mathrm{AA}^{\prime} \mathrm{MnH}_{6}$ and $\mathrm{NaYRuH}_{6}$ have lower specific thermal capacity $\left(C_{v}\right)$ relative to other $\mathrm{AA}^{\prime} \mathrm{MH}_{6}$ materials. The highest $C_{v}$ at $300 \mathrm{~K}$ found in this work is from $\mathrm{BaSrZnH}_{6}\left(127.96 \mathrm{JK}^{-1} \mathrm{~mol}^{-1}\right)$. Moreover, our band structure calculations show that 5 of $\mathrm{AA}^{\prime} \mathrm{MH}_{6}$ materials are wide-bandgap semiconductors, and 11 of them are indirect semiconductors.

\section{Method}

\subsection{Generative Adversarial Network}

The hypothetical materials used in our research are generated by our CubicGAN [22], a generative adversarial network (GAN) based model for generating cubic crystal structures in a high-throughput manner. Our GAN model consists of a generator network and a discriminator/critic network. The discriminator learns to tell real materials from fake materials generated by the generator. The generator learns how to generate samples with similar distribution as the training samples. After trained, we can sample from the generator to generate new materials. In CubicGAN, we focused on generating ternary and quaternary materials with the space groups 221, 225, and 216. Moreover, to simplify the problem, CubicGAN uses special fractional coordinates, all in the set of $\{0.0,0.25,0.5,0.75\}$. The CubicGAN is trained using material data from OQMD [23, 24] and is evaluated on material data from Materials Project [25] and ICSD [26]. The main framework of CubicGAN and the post-processing for the generated materials are shown in Figure 1. It is notoriously hard to train the original GAN model because the adversarial loss is not continuous in the generator, which causes vanishing gradients and saturation in the discriminator. We take advantage of the Wasserstein GAN with gradient penalty by penalizing the norm of gradients of the critic with respect to the inputs [18]. The critic takes real materials and fake materials generated by the generator and then outputs a score which can be interpreted as 
how real the input materials are. The score is used to update the parameters of models of the generator and the critic. The adversarial loss is defined as:

$$
\mathcal{L}=\underset{\tilde{\mathbf{x}} \sim \mathbb{P}_{g}}{\mathbb{E}}[D(\tilde{\mathbf{x}})]-\underset{\mathbf{x} \sim \mathbb{P}_{r}}{\mathbb{E}}[D(\mathbf{x})]+\lambda \underset{\hat{\mathbf{x}} \sim \mathbb{P}_{\hat{\mathbf{x}}}}{E}\left[\left(\left\|\nabla_{\hat{\mathbf{x}}} D(\hat{\mathbf{x}})\right\|_{2}-1\right)^{2}\right]
$$

where $\hat{\mathbf{x}}$ is the linear interpolation between a real material and the generated one and $\underset{\hat{\mathbf{x}} \sim \mathbb{P}_{\hat{\mathbf{x}}}}{E}\left[\left(\left\|\nabla_{\hat{\mathbf{x}}} D(\hat{\mathbf{x}})\right\|_{2}-1\right)^{2}\right]$ is the gradient penalty which enforces gradients with norm at most 1 everywhere. $\lambda$ is set 10 in this work.

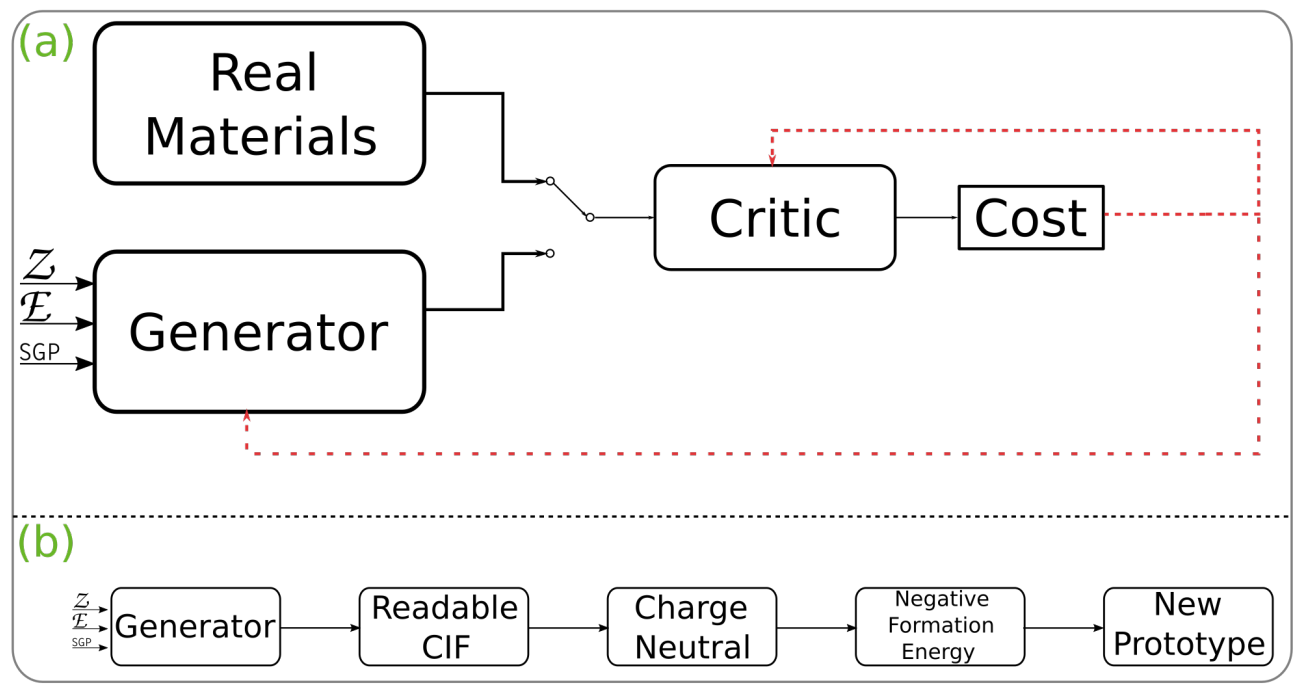

Figure 1: The main framework of CubicGAN. (a) WGAN architecture and (b) post-processing of generated samples.

Conditioning on random noise, three or four-element combinations, and space group, the generator not only generates novel materials with existing prototypes but also generates novel stable ones with new prototypes. When the CubicGAN generates 10 million materials, it can rediscover most of the cubic materials in Materials Project and ICSD. In CubicGAN, we only focus on the generated materials with new prototypes, which are defined by the anonymous formula and the space group ID. In total, 24 and 1 new prototypes are found in 10 million generated ternary and quaternary materials, respectively. Sub-figure (a) of Figure 1 shows how to filter out the materials. On average, $90 \%$ of generated materials have readable CIFs, and we only select materials with neural charge and negative formation energy predicted by CGCNN [27]. After filtering down materials with novel prototypes, we performed DFT calculations, and 36847 candidate materials have been relaxed successfully. Further, 506 stable materials are verified by phonon dispersion.

\subsection{Semiconductor - Metal Classifier}

Table 1: The total number $(N)$ and percentage of ternary and quaternary non-metals (NM) and metals (M) for all the types of Bravais lattice and also for Cubic structures (Cubic-NM and Cubic-M) in the Material project database [28].

\begin{tabular}{|c|c|c|c|c|c|c|c|c|}
\hline Material & $N_{\mathrm{NM}}$ & $N_{\mathrm{M}}$ & $\mathrm{NM} \%$ & $\mathrm{M} \%$ & $N_{\text {Cubic-NM }}$ & $N_{\text {Cubic-M }}$ & Cubic-NM \% & Cubic-M \% \\
\hline Ternary & 28102 & 35682 & 44.06 & 55.94 & 2094 & 8385 & 19.98 & 80.02 \\
\hline Quaternary & 28527 & 10497 & 73.10 & 26.90 & 2578 & 1438 & 64.19 & 35.81 \\
\hline
\end{tabular}

As the CubicGAN model generates only ternary and quaternary materials, we first analyzed the number of non-metals (semiconductors and insulators), and metals in the material project (MP) database [28], as shown in Table 1. We collected all quaternary materials where the bandgap details are available using Pymatgen code [29]. It could be found that $\approx 44 \%$ of the ternary materials are non-zero bandgap materials while $\approx 56 \%$ are metals. However, $\approx 73 \%$ of the quaternary materials are semiconductors or insulators, whereas only $\approx 27 \%$ of them are metals. This indicates that the probability of finding a stable quaternary material with a non-zero bandgap is higher compared to finding that in a ternary material set. We also compared the same details of the cubic materials. It is interesting that $\approx 80 \%$ of the cubic ternary materials are metals, and only $\approx 20 \%$ of them are non-metals. On the contrary, the quaternary cubic materials have $30 \%$ more non-zero bandgap materials than the number of metals. It shows that there is a low probability of 
discovering a non-zero bandgap cubic ternary compound. Instead, in this project, we mainly focused on the quaternary cubic materials for finding stable semiconductors. In this way, by reducing the search space of the materials, we are able to shorten the computational time taken by the DFT calculations.

To develop a semiconductor-metal classifier, we first collected the pretty formulas, Bravais lattice type, and bandgap details of all the quaternary materials from the MP database. There were 28527 non-zero bandgap materials (semiconductors and insulators) and 10497 metals in the collected dataset. In order to let our machine learning models learn the hidden information of both classes of the materials fairly, we randomly selected 10497 number of non-zero bandgap materials. Thus, both metal and non-metal classes have an equal number of components.

We first transformed the Bravais lattice type of the materials into one-hot-encoding. We considered 55 elemental and electronic structure attributes, such as the first ionization energy, atomic volume, electronegativity, total number of valence electrons, and number of valence electrons in s, p, d, and f orbitals, to develop the feature set (see Supporting Information). The weighted average (Avg.) and a maximum difference of those properties for a given chemical formula were added to the feature set. The Avg. of a property $S$ of a quanternary compound $\mathrm{A}_{\alpha} \mathrm{B}_{\beta} \mathrm{C}_{\gamma} \mathrm{D}_{\delta}$ was calculated based on the following expression,

$$
S_{\mathrm{A}_{\alpha} \mathrm{B}_{\beta} \mathrm{C}_{\gamma} \mathrm{D}_{\delta}}^{A v g}=\frac{1}{\alpha+\beta+\gamma+\delta}\left(\alpha S_{A}+\beta S_{B}+\gamma S_{C}+\delta S_{D}\right),
$$

where $S_{\mathrm{A}}, S_{\mathrm{B}}, S_{\mathrm{C}}$ and $S_{\mathrm{D}}$ are the property $\mathrm{S}$ of A, B, C, and D elements, respectively. We also encoded the composition based on a vector with 117 components, where each component represents an element in the periodic table. Altogether, 226 features were considered for training the models.

To create the classifier, we developed a DNN model with two hidden layers using Keras [30] on top of TensorFlow [31]. The first and second hidden layers of DNN include 200, and 100 neurons, respectively. In order to include the nonlinearity into the system, we shifted the summed weighted inputs of each layer through the rectified linear unit $(\mathrm{ReLu})$ activation function. We randomly dropped out $5 \%$ of the units of the hidden layers while training the models. This process is very important for limiting the overfitting of training data. Another useful approach to diminishing overfitting is weight regularization. We employed Ridge (L2) regularization method for adding penalties during updating weights. The adaptive moment estimation (Adam) optimizer with a 0.001 learning rate was considered with binary cross-entropy as the loss function and the metric during the calculations. The optimized number of epochs and batch size are 500 and 1500 , respectively.

To understand the most influential elemental and electronic attributes to classify a material as non-metal or metal, we studied the feature importance (FI) of the materials using a random forest classifier (RFC). Here, we removed the composition vector which was used in the DNN model since we want only the elemental and electronic attributes in the feature importance. We created RFC based on the Scikit-learn code [32] by considering decision trees, minimum samples split, minimum samples leaf, maximum features, and the maximum depth, as 1000, 6, 2, 4, and 90, respectively. The classification report of the RFC model is mentioned in the Supporting Information.

\subsection{Density Functional Theory Calculations}

Density functional theory calculations were performed as implemented in the Vienna ab simulation package (VASP) code [33, 34, 35, 36]. The electron wave functions were described using the PAW pseudopotentials [37, 38]. The exchange-correlation interactions were treated based on the generalized gradient approximation (GGA) within the Perdew-Burke-Ernzerhof (PBE) formulation [39, 40]. The energy threshold value of the plane-wave basis was set as $500 \mathrm{eV}$. In addition, the energy convergence criteria were set to $10^{-8} \mathrm{eV}$, and the force convergence criterion for the ionic steps is set to $10^{-2} \mathrm{eV}$. The Brillouin zone integrations were performed using a dense k-point mesh within the Monkhorst-Pack scheme for the structure optimizations, band structure, density of states, mechanical properties, and phonon calculations. Phonon dispersions were obtained using phonopy code [41]. The elastic constants were calculated by employing density functional perturbation theory (DFPT) as implemented in VASP [42]. VASPKIT code [43] was used to obtain the bulk modulus (K), Shear modulus (G), Young's modulus (Y), and Poisson's ratio $(\mu)$ of the materials based on Hill method [44].

\section{Results and Discussion}

\subsection{Predicting New Semiconductors}

We studied FI using the RFC model. Even though both Avg. and the maximum difference of each atomic/electronic property were considered for the RFC model, only four features related to maximum difference have FI greater than 1 $\%$. This indicates that Avg. of the properties plays a significant role when classifying a material like metal or non-metal. 
The top 10 features are mentioned in Fig. 2. Avg. Availability of metal and transition-metal atoms have high FI, indicating having metallic atoms is important for the material to be a metal or a semiconductor/insulator. The Avg. number of $\mathrm{p}\left(\mathrm{Np}_{\mathrm{val}}\right)$ and $\mathrm{d}\left(\mathrm{Nd}_{\mathrm{val}}\right)$ valence electrons are the two electronic properties in the top 10 features. The thermodynamical properties like average atomic boiling point and atomic specific heat capacity also have high FI percentages. When the radius of the atom is high, electrons are less attracted to the nuclei. Therefore, the electrons can behave as free electrons for providing metallic properties. When more valence electrons exist with a high atomic radius, there is a high probability that those elements can contribute to forming a metallic character in a material. Thus, low atomic density will be preferred for metals. This can be the main reason for having high FI for Avg. Atomic density, volume, and covalent radius.

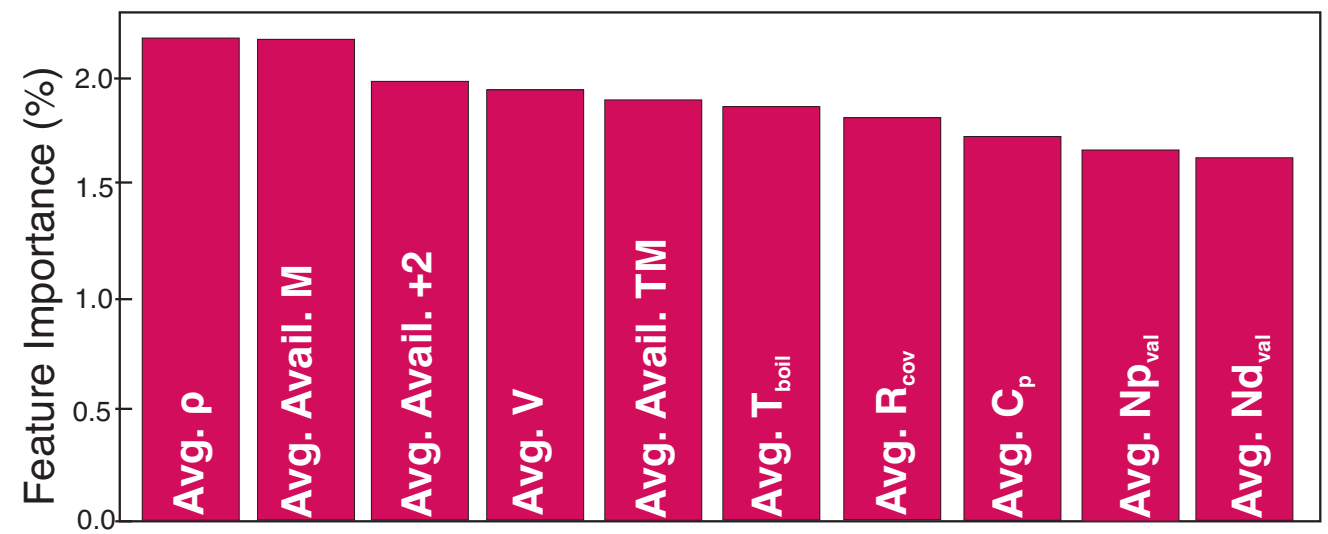

Figure 2: Feature Importance as a percentage from the RFC model. Labels on the x-axis: atomic density ( $\rho$ ), metal atom $(\mathrm{M}),+2$ oxidation state $(+2)$, transition metal atom $(\mathrm{TM})$, atomic volume $(\mathrm{V})$, boilling point $\left(\mathrm{T}_{\text {boil }}\right)$, covalent radius $\left(\mathrm{R}_{\text {cov }}\right)$, specific heat capacity at constant pressure $\left(\mathrm{C}_{p}\right)$, number of $\mathrm{p}$-valence electrons $\left(\mathrm{Np}_{\mathrm{val}}\right)$ and number of d-valence electrons $\left(\mathrm{Nd}_{\mathrm{val}}\right)$. Avg. and Avail. stands for weighted average and availability, respectively.
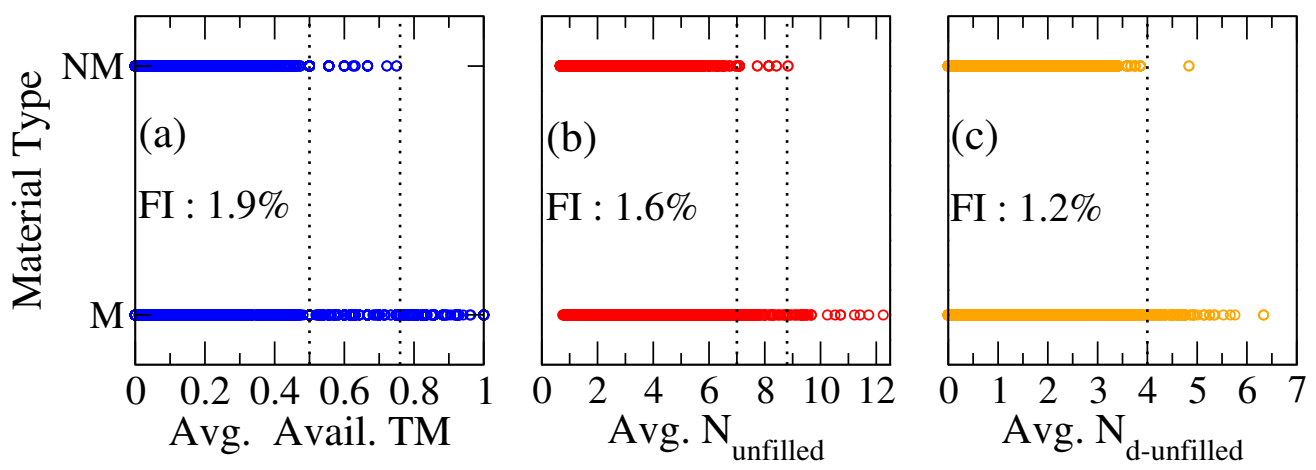

Figure 3: Material type (NM: Non-metal, M: Metal) as a function of (a) weighted average (Avg.) of availability (Avail.) of transition metal (TM) $(\rho)$, (b) Avg. of number of unfilled valence orbitals $\left(\mathrm{N}_{\text {unfilled }}\right)$, and (c) Number of unfilled valence d-orbitals $\left(\mathrm{Ne}_{\mathrm{d} \text {-unfilled }}\right)$. Feature Importance $(\mathrm{FI})$ of each attribute is also mentioned as a percentage.

We further analyzed the descriptors with FI percentage in the range of 1 to $2 \%$ by plotting non-metal and metal classes of all the 39024 quaternary materials against the descriptors. As can be seen in Figure 3 , an elemental property and two electronic properties show interesting behavior. Those properties are the Avg. Availability of elements from d-block (transition metal), the Avg. Number of unfilled valence orbitals and the Avg. Number of unfilled d-valence orbitals. The availability of d-block elements for a given chemical formula is 1 when all the elements transition metal elements. As shown in Fig. 3 (a), the number of non-metals is decreasing since 0.4, implying the probability of finding a semiconductor or an insulator decreases after this value. Within the 39024 quaternary materials, there was no semiconductor or insulator after $0.76 \mathrm{Avg}$. Availability of transition metal elements. After Avg. Number of unfilled valence orbitals is 7, probability of finding a non-metal becomes low (see Fig. 3 (b)). Avg. Number of unfilled d valence orbitals also has a critical value, which is around 4 (see Fig. 3(c)). This will be helpful for one to restrict their chemical space while searching semiconductors with transition metal elements. 
Figure 4 shows the normalized confusion matrix for the DNN classifier. It is apparent that $41 \%$ of the instances were classified as true metals while $43 \%$ of the materials were listed as true non-metals. The percentages of false metals and false non-metals were $9.8 \%$ and $6 \%$. Thus, we can expect an approximately balanced binary classification from the trained model. The classification report for the DNN model is shown in Table 2. It is clear that the DNN classifier predicts whether a quaternary material is a metal or non-metal with 0.85 accuracies. Precision is the matrix that compares the number of true positive instances with the number of predicted positive instances. In our work, the DNN model classifies a material as a non-metal with 0.82 and metal with 0.88 precision. The recall is a measure of the number of correctly predicted positive cases compared to the total number of positive cases in the dataset. Table 2 shows that there is 0.88 recall for non-metal, while there is 0.82 recall for metals. By combining precision and recall, F1-score can be calculated as 0.85 for both metal and non-metal classes.

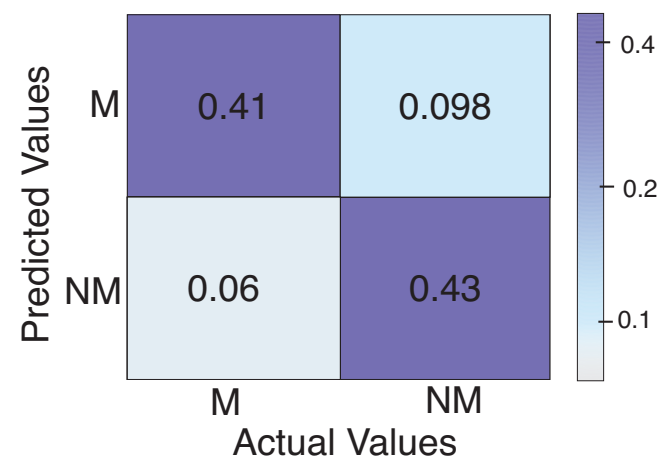

Figure 4: Normalized confusion matrix of semiconductor-metal classifier for metals (M) and non-metals (NM).

Table 2: The classification report of the test set for the semiconductor - metal classifier

$\begin{array}{lllll} & \text { precision } & \text { recall } & \text { f1-score } & \text { support } \\ \text { Metal } & 0.88 & 0.82 & 0.85 & 1800 \\ \text { Non-metal } & 0.82 & 0.88 & 0.85 & 1698 \\ \text { accuracy } & & & 0.85 & 3498 \\ \text { macro avg } & 0.85 & 0.85 & 0.85 & 3498 \\ \text { weighted avg } & 0.85 & 0.85 & 0.85 & 3498\end{array}$

To show the methodology of finding stable semiconductors based on generative adversarial networks, we applied our DNN classifier on CubicGAN predicted mechanically and dynamically stable quaternary materials. Out of 323 quaternary new materials predicted by the CubicGAN model, 101 compounds were classified as non-metals by our binary classifier.

\subsection{Structure and Thermodynamic Stability}

We carried out our DFT calculations on those non-metals to find thermodynamically stable semiconductors. We discovered that 12 semiconductors, which have chemical formulas in the form of $\mathrm{AA}^{\prime} \mathrm{MH}_{6}$, exhibit zero energy-abovehull against the respective competing phases. Those are $\mathrm{BaNaRhH}_{6}, \mathrm{BaSrZnH}_{6}, \mathrm{BaCsAlH}_{6}, \mathrm{SrTlIrH}_{6}, \mathrm{KNaNiH}_{6}$, $\mathrm{NaYRuH}_{6}, \mathrm{CsKSiH}_{6}, \mathrm{CaScMnH}_{6}, \mathrm{YZnMnH}_{6}, \mathrm{NaZrMnH}_{6}, \mathrm{AgZrMnH}_{6}, \mathrm{AgZrMnH}_{6}$, and ScZnMnH $\mathrm{SHe}_{6}$ also find that Kadir et al. reported 5 different $\mathrm{AA}^{\prime} \mathrm{MH}_{6}$ type semiconductors, where $\mathrm{M}=\mathrm{Ir}$ [45]. They were able to synthesize $\mathrm{NaCaIrH}_{6}, \mathrm{NaSrIrH}_{6}, \mathrm{NaBaIrH}_{6}, \mathrm{KSrIrH}_{6}$, and $\mathrm{KBaIrH}_{6}$ by direct combination of the alkali (Na and K), alkaline earth $(\mathrm{Ca}, \mathrm{Ba}$, and $\mathrm{Sr})$ binary hydrides/deuterides with Ir powder. Their X-ray and neutron powder diffraction studies confirm that those semiconductors have the space group symmetry F-43m. Furthermore, open quantum materials database (OQMD) [46, 47] contains the structural properties and band gaps of $\mathrm{NaCaIrH}_{6}, \mathrm{NaSrIrH}_{6}, \mathrm{NaBaIrH}_{6}$ semiconductors and the MP database has those information of $\mathrm{NaCaIrH}_{6}$, and $\mathrm{NaBaIrH}_{6}$ semiconductors [28] (See Supporting Information).

CubicGAN generates conventional structures with cubic Bravais lattice with F-43m (216) space group for $\mathrm{AA}^{\prime} \mathrm{MH}_{6}$ materials, which have 36 atoms. On the contrary, the primitive unit cell with hexagonal Bravais lattice has only 9 atoms. Therefore, we considered the hexagonal unit cell to lower the computational time of the DFT calculations. In the primitive unit cells (see Fig 5), green and red sites are symmetrically equivalent, while grey sites are located at the right middle of the hexagonal unit cell. Thus, we label green and red sites as $\mathrm{A}$ and $\mathrm{A}^{\prime}$, while the middle site is $\mathrm{M}$. Rest of the 6 sites are occupied by $\mathrm{H}$ atoms. In the research work of Kadir et al., they considered alkali atoms as A atoms, 

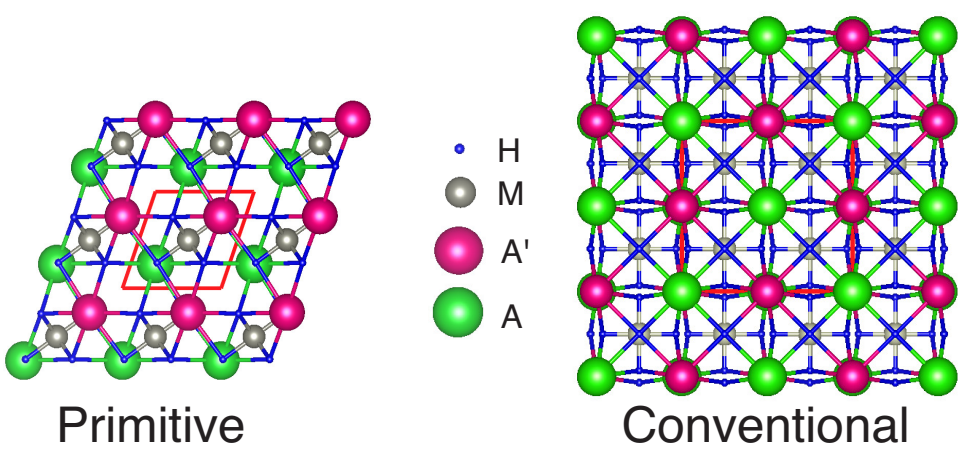

Figure 5: Side view of the structure of $\mathrm{AA}^{\prime} \mathrm{MH}_{6}$ materials with primitive and conventional unitcells, which are indicated by red lines.

alkaline earth atoms as $\mathrm{A}^{\prime}$ atoms, and $\mathrm{M}$ atoms as Ir. In this research, our findings show that both $\mathrm{A}$ and $\mathrm{A}^{\prime}$ atoms can be alkali atoms (E.g., $\mathrm{CsKSiH}_{6}$ ) or alkaline earth atoms (E.g., $\mathrm{BaSrZnH}_{6}$ ). Moreover, the $\mathrm{M}$ atom can be a transition metal atom or even $\mathrm{Al}$ or $\mathrm{Si}$. Therefore, our experiments show that those materials can have high chemical diversity.

The lattice parameters, A-H, M-H, A-M, and A-A' bond lengths, are mentioned in Table 3 . The primitive hexagonal unit cells have $a / c=1$ lattice parameter ratio making $a=b=c$. As shown in Table 3 , Mn-related $\mathrm{AA}^{\prime} \mathrm{MH}_{6}$ and $\mathrm{NaYRuH}_{6}$ structures have the shortest lattice parameters compared to the rest of the materials. They have lattice parameters less than $5.0 \AA$, while other materials have greater than $5.4 \AA$. All $\mathrm{A}, \mathrm{A}^{\prime}$ and $\mathrm{M}$ elements make bonds with $\mathrm{H}$ atoms. $\mathrm{A}$ and $\mathrm{A}^{\prime}$ elements are bonded to twelve equivalent $\mathrm{H}$ atoms to form $\mathrm{AH}_{12}$ and $\mathrm{A}^{\prime} \mathrm{H}_{12}$ cuboctahedra. And also, $\mathrm{M}$ atoms make $\mathrm{MH}_{6}$ octahedra by making bonds with $6 \mathrm{H}$ atoms. $\mathrm{An} \mathrm{AH}_{12}\left(\mathrm{~A}^{\prime} \mathrm{H}_{12}\right)$ cuboctahedra shares corners with twelve equivalent $\mathrm{AH}_{12}\left(\mathrm{~A}^{\prime} \mathrm{H}_{12}\right)$ cuboctahedra. Moreover, they share faces with four $\mathrm{MH}_{6}$ octahedra [48]. Due to symmetry, A-H and $\mathrm{A}^{\prime}-\mathrm{H}$ bond lengths are equal. $\mathrm{M}-\mathrm{H}$ bond lengths are the shortest compared to other bonds for a given compound. A- $\mathrm{A}^{\prime}$ of Mn-related $\mathrm{AA}^{\prime} \mathrm{MH}_{6}$ and $\mathrm{NaYRuH}_{6}$ structures are less than $3.4 \AA$, and $\mathrm{A}-\mathrm{M}$ and $\mathrm{A}^{\prime}$-M distances are less than 3.1 $\AA$. It can cause strong interactions between those atoms. A- $\mathrm{A}^{\prime}$ distance for the rest of the materials is greater than $3.8 \AA$, and $\mathrm{A}-\mathrm{M}$ and $\mathrm{A}^{\prime}-\mathrm{M}$ distances are greater than $3.3 \AA$, indicating relatively weaker interactions.

The thermodynamical stability of the $\mathrm{AA}^{\prime} \mathrm{MH}_{6}$ materials against their elements was studied using the formation energies, which were based on the following equation.

$$
E \_ \text {form }=\frac{1}{N}\left(E_{\mathrm{tot}}-x_{i} \sum_{i} E_{i}\right)
$$

Here, $E_{\text {tot }}$ is the total energy per unit formula of the material. $x_{i}$ is the number of atoms of each element in the unit formula; i.e., 1 for $\mathrm{A}, \mathrm{A}^{\prime}, \mathrm{M}$ atoms and 6 for $\mathrm{H} . N=\sum x_{i}$; i.e., 9 for $\mathrm{AA}^{\prime} \mathrm{MH}_{6}$. To find the atomic energies $\left(E_{i}\right)$, we collected the most stable structures of each element using Pymatgen code [29]. Same DFT settings were used to calculate the energy of each element. It is clear that all the six materials have negative formation energies, which confirms their stability.

\subsection{Mechanical Properties and Stability}

Next, we studied the mechanical properties and stability of the $\mathrm{AA}^{\prime} \mathrm{MH}_{6}$ materials by calculating the elastic constants using the DFPT method. To analyse the mechanical properties, we used the Vaspkit code [43], where it computes the elastic constants by considering the $\mathrm{AA}^{\prime} \mathrm{MH}_{6}$ cubic system. Since cubic unitcells has $a=b=c$ lattice lengths and $\alpha=\beta=\gamma=90^{0}$ lattice angles, $C_{11}=C_{22}=C_{33}, C_{44}=C_{55}=C_{66}$, and $C_{12}=C_{13}=C_{23}$ [49]. Therefore, we mentioning only the three independent elastic constants $\left(C_{11}, C_{12}\right.$ and $\left.C_{44}\right)$ in Table 4 . It is clear that $\mathrm{AA}^{\prime} \mathrm{MH}_{6}$ materials have relatively higher $C_{11}$ for $\mathrm{AA}^{\prime} \mathrm{MnH}_{6}$ and $\mathrm{NaYRuH}_{6}$, compared to the other four materials in Table 4 . As discussed before, the lattice constants, and $\mathrm{A}-\mathrm{A}^{\prime}$ bond lengths of $\mathrm{AA}^{\prime} \mathrm{MnH}_{6}$ and $\mathrm{NaYRuH}_{6}$ structures are considerably lower than that of the rest of the materials. As illustrated by Fig. 5. A-A' bonds are aligned in $\mathbf{a}, \mathbf{b}$ and $\mathbf{c}$ directions. $C_{11}, C_{22}$, and $C_{33}$ are parallel to $\mathbf{a}, \mathbf{b}$ and $\mathbf{c}$ directions, respectively. Therefore, higher $C_{i i}(\mathrm{i}=1,2$ and 3$)$ can be mainly due to the strong interactions between the $\mathrm{A}$ and $\mathrm{A}^{\prime}$ atoms. Born stability criteria for the cubic systems are $C_{11}-C_{12}>0, C_{11}+2 C_{12}>0$ and $C_{44}>0$ [49]. It is clear from Table 4 that all the eight materials comply with the above requirements. 
Table 3: The lattice parameter $(a)$ and bond lengths in $\AA$, and the formation energies in eV/atom for $\mathrm{AA}^{\prime} \mathrm{MH}_{6}$ materials.

\begin{tabular}{|c|c|c|c|c|c|c|}
\hline Material & $a$ & $\mathrm{~A}-\mathrm{H}$ & $\mathrm{M}-\mathrm{H}$ & $\mathrm{A}-\mathrm{M}$ & $\mathrm{A}^{\prime} \mathrm{A}^{\prime}$ & $E_{\text {form }}$ \\
\hline BaNaRhH $_{6}$ & 5.5105 & 2.76924 & 1.67023 & 3.37448 & 3.89651 & -0.4678 \\
\hline $\mathrm{BaSrZnH}_{6}$ & 5.47201 & 2.73801 & 1.83004 & 3.35091 & 3.8693 & -0.3496 \\
\hline $\mathrm{BaCsAlH}_{6}$ & 5.84781 & 2.93934 & 1.76669 & 3.58104 & 4.13503 & -0.3159 \\
\hline $\mathrm{SrTlIrH}_{6}$ & 5.56492 & 2.79622 & 1.69039 & 3.4078 & 3.93499 & -0.3488 \\
\hline $\mathrm{CsKSiH}_{6}$ & 5.86576 & 2.968 & 1.61863 & 3.59203 & 4.14772 & -0.1817 \\
\hline $\mathrm{KNaNiH}_{6}$ & 5.40252 & 2.73044 & 1.51196 & 3.30835 & 3.82016 & -0.1715 \\
\hline $\mathrm{NaYRuH}_{6}$ & 4.97064 & 2.48578 & 1.70949 & 3.04388 & 3.04388 & -0.4999 \\
\hline $\mathrm{CaScMnH}_{6}$ & 4.74883 & 2.37467 & 1.64383 & 2.90805 & 3.35793 & -0.5261 \\
\hline $\mathrm{YZnMnH}_{6}$ & 4.69455 & 2.34755 & 1.62362 & 2.87481 & 3.31955 & -0.3099 \\
\hline $\mathrm{NaZrMnH}_{6}$ & 4.64255 & 2.32129 & 1.63348 & 2.84297 & 3.28278 & -0.3111 \\
\hline $\mathrm{AgZrMnH}_{6}$ & 4.62246 & 2.31127 & 1.62077 & 2.83067 & 3.26857 & -0.2070 \\
\hline $\mathrm{ScZnMnH}_{6}$ & 4.50635 & 2.25322 & 1.60682 & 2.75957 & 3.18647 & -0.3070 \\
\hline
\end{tabular}

We also calculated the Bulk modulus $(K)$, Young's modulus $(Y)$, and isotropic Poisson's ratio $(\mu)$ based on Hill approximation [50] as mentioned in Table 4 . Smallest $K$ values were found from $\mathrm{CsKSiH}_{6}(16.615 \mathrm{GPa})$, while largest value was calculated from $\mathrm{AgZrMnH}_{6}$ (120.755 GPa). $\mathrm{SrTlIrH}_{6}$ (21.915 GPa) provides lowest $Y$, while $\mathrm{NaZrMnH}_{6}$ $\left(156.876 \mathrm{GPa}\right.$ ) exhibits the maximum $Y$. It is clear that $\mathrm{NaYRuH}_{6}$ and all the Mn-based materials have significantly larger $K$ and $Y$ values than that of the other six materials. This can be mainly because of high $C_{i i}(\mathrm{i}=1,2$, and 3$)$ formed due to strong A-A' bonds. Because of low $Y, \mathrm{NaYRuH}_{6}$ and $\mathrm{Mn}$-based $\mathrm{AA}^{\prime} \mathrm{MH}_{6}$ materials can be considered stiffer materials relative to the other six semiconductors. And also, they exhibit more resistance to compression due to high $K$. All the $\mu$ values of the $\mathrm{AA}^{\prime} \mathrm{MH}_{6}$ materials are between 0.2 and 0.4 . maximum $\mu$ was found from $\mathrm{SrTlIrH}_{6}$. Thus, $\mathrm{SrTIIHH}_{6}$ has considerably low $Y$ and high $\mu$. This indicates that $\mathrm{SrTlIrH}_{6}$ semiconductor is less stiff due to small $Y$ and more deformable elastically at small strains due to large $\mu$.

Table 4: The mechanical properties of the $\mathrm{AA}^{\prime} \mathrm{MH}_{6}$ materials. The $C_{11}, C_{12}, C_{44}$ elastic constants, bulk modulus $(K)$, Shear modulus $(G)$ and Young's modulus $(Y)$ were calculated in GPa. $\mu$ is the isotropic Poisson's ratio.

\begin{tabular}{|c|c|c|c|c|c|c|c|}
\hline Material & $C_{11}$ & $C_{12}$ & $C_{44}$ & $K$ & $G$ & $Y$ & $\mu$ \\
\hline BaNaRhH6 & 60.514 & 20.174 & 11.901 & 33.621 & 14.722 & 38.541 & 0.309 \\
\hline BaSrZnH6 & 86.238 & 20.648 & 30.492 & 42.512 & 31.393 & 75.577 & 0.204 \\
\hline BaCsAlH6 & 50.455 & 16.174 & 13.119 & 27.601 & 14.602 & 37.24 & 0.275 \\
\hline SrTIIrH6 & 52.588 & 26.735 & 5.549 & 35.353 & 7.845 & 21.915 & 0.397 \\
\hline CsKSiH6 & 28.939 & 10.454 & 7.203 & 16.615 & 7.960 & 20.592 & 0.293 \\
\hline KNaNiH6 & 36.027 & 11.720 & 6.618 & 19.822 & 8.463 & 22.225 & 0.313 \\
\hline NaYRuH6 & 131.450 & 36.248 & 44.149 & 67.982 & 45.499 & 111.600 & 0.226 \\
\hline CaScMnH6 & 192.385 & 28.606 & 38.997 & 83.199 & 52.744 & 130.627 & 0.238 \\
\hline YZnMnH6 & 171.063 & 50.92 & 23.563 & 90.968 & 34.649 & 137.167 & 0.331 \\
\hline NaZrMnH6 & 196.714 & 47.003 & 57.275 & 96.907 & 63.761 & 156.876 & 0.23 \\
\hline AgZrMnH6 & 190.957 & 85.654 & 50.274 & 120.755 & 51.211 & 134.606 & 0.314 \\
\hline ScZnMnH6 & 191.749 & 60.602 & 60.537 & 104.317 & 62.504 & 156.295 & 0.250 \\
\hline
\end{tabular}

\subsection{Thermodynamic Properties and Dynamical Stability}

The temperature of the highest normal mode of a crystal is known as the Debye temperature $\theta_{D}$. This can be obtained by employing Debye sound velocity $\left(\nu_{D}\right)$ as explained by Eq. 4. Debye sound velocity can be calculated using the longitudinal and transverse sound velocities, which can be determined based on $K$ and $G$ as shown in Eq. 66 [51]. Here, $N, V_{0}$, and $\rho$ are the number of atoms, volume, and density of the unicell, respectively. And also, $h$ is Plank's constant, and $k_{B}$ is Boltzmann's constant.

$$
\begin{gathered}
\theta_{D}=\frac{h}{k_{B}}\left(\frac{3 N}{4 \pi V_{0}}\right)^{\frac{1}{3}} \nu_{D} \\
\nu_{D}=\left[\frac{1}{3}\left(\frac{2}{\nu_{l}^{3}}+\frac{1}{\nu_{t}^{3}}\right)\right]^{-\frac{1}{3}}
\end{gathered}
$$


Generative design of semiconductor materials
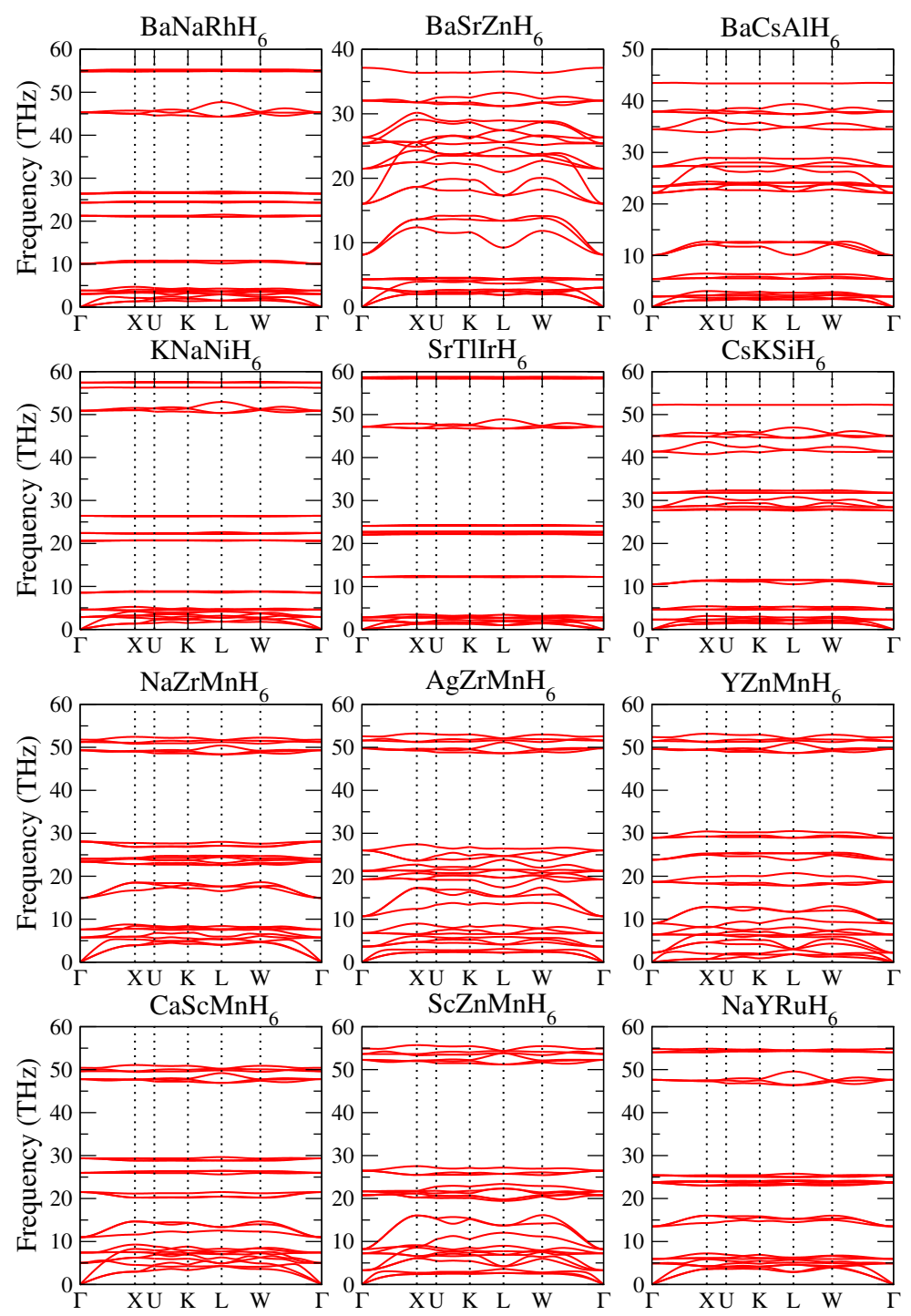

Figure 6: Phonon dispersion of $\mathrm{AA}^{\prime} \mathrm{MH}_{6}$ materials.

$$
\nu_{l}=\left(\frac{3 K+4 G}{3 \rho}\right)^{\frac{1}{2}} \text { and } \nu_{t}=\left(\frac{G}{\rho}\right)^{\frac{1}{2}}
$$

Table 5 shows the respective $\rho, \nu_{l}, \nu_{t}, \nu_{D}$ and $\theta_{D}$ values for $\mathrm{AA}^{\prime} \mathrm{MH}_{6}$ crystals. Debye temperature of $\mathrm{NaYRuH}_{6}$ and Mn-based $\mathrm{AA}^{\prime} \mathrm{MH}_{6}$ materials are significantly higher than that of other $\mathrm{AA}^{\prime} \mathrm{MH}_{6}$ materials. As $\theta_{D}$ depends on $K$ and $G$ (see Eq. 6 and 4), enhanced $\theta_{D}$ is due to high $K$ and $G$ of those semiconductors.

We also plotted $C_{v}$ as a function of temperature $T$ using the Phonopy code [41]. $C_{v}$ can be determined based on the following expression,

$$
C_{v}=\sum_{\mathbf{q} j} k_{B}\left(\frac{\hbar \omega_{\mathbf{q} j}}{k_{B} T}\right)^{2} \frac{\exp \left(\hbar \omega_{\mathbf{q} j} / k_{B} T\right)}{\left[\exp \left(\hbar \omega_{\mathbf{q} j} / k_{B} T\right)-1\right]^{2}},
$$

where $\omega_{\mathbf{q} j}$ is the phonon frequency for $\mathbf{q}$ wave vector at $j$ th phonon band index and $\hbar$ is the reduced Plank's constant [41]. As can be seen in Fig 7, $C_{v}$ of $\mathrm{NaYRuH}_{6}$ and Mn-based $\mathrm{AA}^{\prime} \mathrm{MH}_{6}$ materials are plotted with broken lines, and that of the rest of the materials are indicated by solid lines. It is clear that $C_{v}$ of $\mathrm{NaYRuH}_{6}$ and $\mathrm{Mn}^{-b a s e d ~} \mathrm{AA}^{\prime} \mathrm{MH}_{6}$ materials are smaller than that of the other materials at the low temperatures ( 0 to $150 \mathrm{~K})$. At the low temperature limit 
Generative design of semiconductor materials

( $\left.T \geq \theta_{D}, \theta_{D} / T \ll 1\right), C_{v}$ is proportional to $\left(T / \theta_{D}\right)^{3}$. Since $\theta_{D}$ is higher compared to that of other materials, $C_{v}$ is smaller at low temperatures for $\mathrm{NaYRuH}_{6}$ and $\mathrm{Mn}$-based $\mathrm{AA}^{\prime} \mathrm{MH}_{6}$.

Table 5: The density $(\rho)$, longitudinal $\left(\nu_{l}\right)$, transverse $\left(\nu_{t}\right)$ and average $\left(\nu_{\mathrm{D}}\right)$ sound velocity, Debye temperature $\left(\theta_{\mathrm{D}}\right)$ and specific thermal capacity at $300 \mathrm{~K}\left(C_{v}^{300 \mathrm{~K}}\right)$ for the $\mathrm{AA}^{\prime} \mathrm{MH}_{6}$ materials.

\begin{tabular}{|l|l|l|l|l|l|c|}
\hline Material & $\rho\left(\mathrm{gcm}^{-3}\right)$ & $\nu_{l}\left(\mathrm{~ms}^{-1}\right)$ & $\nu_{t}\left(\mathrm{~ms}^{-1}\right)$ & $\nu_{\mathrm{D}}\left(\mathrm{ms}^{-1}\right)$ & $\theta_{\mathrm{D}}(\mathrm{K})$ & $C_{v}^{300 \mathrm{~K}}\left(\mathrm{JK}^{-1} \mathrm{~mol}^{-1}\right)$ \\
\hline BaNaRhH$_{6}$ & 4.0401 & 3630.4733 & 6902.4643 & 2858.5852 & 337.0342 & 119.113 \\
\hline BaSrZnH6 $_{6}$ & 3.4683 & 4932.0885 & 12405.1302 & 3901.0279 & 463.1759 & 127.961 \\
\hline BaCsAlH $_{6}$ & 2.9078 & 4023.4038 & 7286.8499 & 3164.0609 & 351.5324 & 115.715 \\
\hline SrTIIrH $_{6}$ & 5.4546 & 2898.0880 & 4445.3015 & 2265.8564 & 264.5375 & 120.116 \\
\hline $\mathrm{CsKSiH}_{6}$ & 1.9584 & 3728.7223 & 5212.2198 & 2901.6325 & 321.3897 & 109.520 \\
\hline $\mathrm{KNaNiH}_{6}$ & 1.5423 & 4490.9338 & 5199.8403 & 3445.3146 & 414.3299 & 121.746 \\
\hline $\mathrm{NaYRuH}_{6}$ & 3.4194 & 6133.7278 & 14188.8339 & 4846.6876 & 633.4998 & 112.140 \\
\hline $\mathrm{CaScMnH}_{6}$ & 2.6144 & 7663.0364 & 14886.6869 & 6036.7538 & 825.9060 & 110.588 \\
\hline $\mathrm{YZnMnH}_{6}$ & 3.9895 & 5863.5797 & 10231.3126 & 4606.2522 & 637.4814 & 116.661 \\
\hline $\mathrm{NaZrMnH}_{6}$ & 3.3572 & 7361.3351 & 16649.9772 & 5814.9095 & 813.7671 & 106.507 \\
\hline AgZrMnH$_{6}$ & 5.0489 & 6118.9252 & 12770.7618 & 4827.2738 & 678.4886 & 106.507 \\
\hline ScZnMnH $_{6}$ & 3.5897 & 7230.2461 & 15811.8943 & 5708.4879 & 823.0192 & 115.952 \\
\hline
\end{tabular}

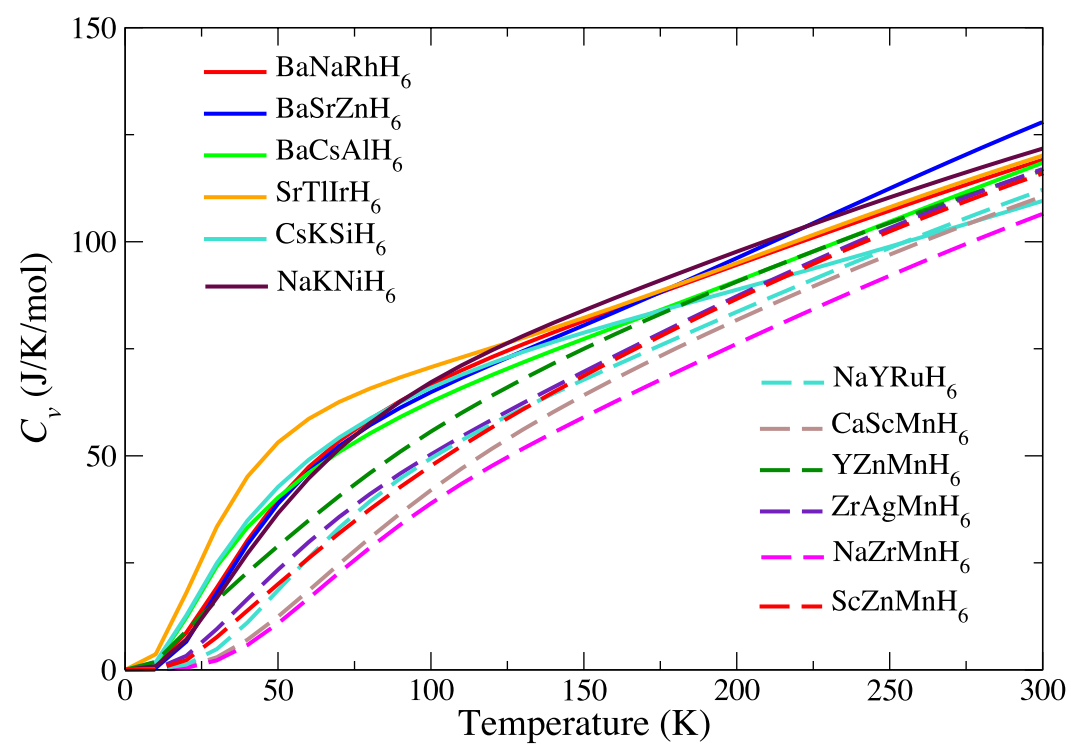

Figure 7: The specific heat capacity $\left(C_{v}\right)$ of $\mathrm{AA}^{\prime} \mathrm{MH}_{6}$ materials as a function of temperature $(T)$.

\subsection{Electronic Properties}

As can be seen in Table 6, A, $\mathrm{A}^{\prime}$ and $\mathrm{M}$ elements lose electrons (except in $\mathrm{Ru}$, where it has small negative value), while $\mathrm{H}$ atoms gain electrons. Thus, we can expect an ionic character in $\mathrm{A}-\mathrm{H}, \mathrm{A}^{\prime}-\mathrm{H}$, and $\mathrm{M}-\mathrm{H}$ bonds. Even though $\mathrm{A}$ and $\mathrm{A}^{\prime}$ sites are symmetrically equivalent, the atoms at those sites can lose a different amount of electrons. This is mainly because atoms at those sites have different oxidation states. Based on Table 6, Na, K, and Cs alkali atoms have their usual oxidation state $(+1)$, while alkaline earth atoms such as $\mathrm{Ca}, \mathrm{Sr}$, and $\mathrm{Ba}$ lose more than 1 electron as they can donate up to 2 electrons. Al, $\mathrm{Si}$, and Tl exhibit their most common oxidation states, which are $+3,+4$, and +1 , respectively. It is reported that first-principles computations provide only negligible changes in the local transition-metal charge for semiconducting crystals [52]. Therefore, we propose that we can consider $\mathrm{MH}_{6}^{n-}$ complex as a single unit since the $\mathrm{M}-\mathrm{H}$ bond lengths are very short compared to other H-related bonds. $n$ can be found by computing $\Delta q_{\mathrm{M}}+6 \times \Delta q_{\mathrm{H}}$, which is greater than 2 for all the $\mathrm{M}$ atoms except for $\mathrm{Ni}$ and $\mathrm{Si}$. For those two atoms, $n \approx 1.6$. Therefore, we can expect $\mathrm{MH}_{6}^{2-}$ for $\mathrm{Si}$ and $\mathrm{Ni}$ complexes, while $\mathrm{MH}_{6}^{3-}$ for the rest of the complexes. Kadir et al. suggest that $\mathrm{IrH}_{6}^{3-}$ complexes exist in $\mathrm{AA}^{\prime} \mathrm{IrH}_{6}$ semiconductors [45]. Therefore, $\mathrm{MH}_{6}^{3-}$ can be the common complex that exists in $\mathrm{AA}^{\prime} \mathrm{MH}_{6} \mathrm{materials}$ 
Generative design of semiconductor materials

Table 6: The Bader charge transfer $(\Delta q)$ in electrons for each element of $\mathrm{AA}^{\prime} \mathrm{MH}_{6}$ Materials.

\begin{tabular}{|l|l|l|l|l|}
\hline Material & $\Delta q_{\mathrm{A}}$ & $\Delta q_{\mathrm{A}^{\prime}}$ & $\Delta q_{\mathrm{M}}$ & $\Delta q_{\mathrm{H}}$ \\
\hline BaNaRhH $_{6}$ & 1.4256 & 0.8613 & 0.0127 & -0.3841 \\
\hline BaSrZnH $_{6}$ & 1.3057 & 1.4588 & 0.6579 & -0.5701 \\
\hline BaCsAlH $_{6}$ & 1.4853 & 0.7135 & 3.0000 & -0.8581 \\
\hline SrTlIrH $_{6}$ & 1.5551 & 0.4674 & 0.2944 & -0.3855 \\
\hline $\mathrm{CsKSiH}_{6}$ & 0.7625 & 0.8483 & 4.0000 & -0.9345 \\
\hline $\mathrm{KNaNiH}_{6}$ & 0.7781 & 0.8734 & 0.5139 & -0.3612 \\
\hline $\mathrm{NaYRuH}_{6}$ & 0.8341 & 1.8845 & -0.1099 & -0.4356 \\
\hline $\mathrm{CaScMnH}_{6}$ & 1.3626 & 1.6427 & 0.2943 & -0.5509 \\
\hline $\mathrm{YZnMnH}_{6}$ & 1.7862 & 0.7053 & 0.3301 & -0.4717 \\
\hline $\mathrm{NaZrMnH}_{6}$ & 0.8179 & 1.8115 & 0.3468 & -0.4978 \\
\hline $\mathrm{AgZrMnH}$ & 0.2876 & 1.8191 & 0.3904 & -0.4168 \\
\hline $\mathrm{ScZnMnH}_{6}$ & 1.6590 & 0.7511 & 0.2937 & -0.4484 \\
\hline
\end{tabular}

Figures 8 and 9 show the band structures and partial density of states (PDOS) of the $\mathrm{AA}^{\prime} \mathrm{MH}_{6}$ materials. It is clear that all the six $\mathrm{AA}^{\prime} \mathrm{MH}_{6}$ materials are semiconductors. The bandgap for each material is mentioned in Table 7. In this research, the highest band gap can be found in $\mathrm{KNaNiH}_{6}(\approx 3.3 \mathrm{eV})$ and the lowest bandgap is in $\mathrm{CaScMnH}_{6}$ $(\approx 1.3 \mathrm{eV})$. The bandgap range of wide-bandgap semiconductors is considered as the range above $2 \mathrm{eV}$ [53]. Thus, $\mathrm{BaNaRhH}_{6}, \mathrm{BaCsAlH}_{6}, \mathrm{CsKSiH}_{6}, \mathrm{KNaNiH}_{6}$, and $\mathrm{NaYRuH}_{6}$ can be identified as wide-bandgap semiconductors. As reported by Kadir et al., $\mathrm{NaCaIrH}_{6}, \mathrm{NaSrIrH}_{6}, \mathrm{NaBaIrH}_{6}, \mathrm{KSrIrH}_{6}$ and $\mathrm{KBaIrH}_{6}$ have bandgaps between 2.91 and $3.33 \mathrm{eV}$ [45] (see Supporting Information). Wide-bandgap semiconductors are vital for manufacturing optical devices emitting green, blue, and UV frequencies and also power devices functioning at higher temperatures [53, 54].

Other than in $\mathrm{BaCsAlH}_{6}$ and $\mathrm{CsKSiH}_{6}$, all the $\mathrm{AA}^{\prime} \mathrm{MH}_{6}$ materials have their conduction band minimum (CBM) at X high-symmetric K-point. The CBM of $\mathrm{BaCsAlH}_{6}$ and $\mathrm{CsKSiH}_{6}$ are at $\Gamma$ points. The valence band maximum (VBM) of $\mathrm{BaNaRhH}_{6}, \mathrm{SrTlIrH}_{6}, \mathrm{YMnZnH}_{6}, \mathrm{NaYRuH}_{6}$, and $\mathrm{AgZrMnH}_{6}$ exist at W K-point. $\mathrm{BaSrZnH}_{6}, \mathrm{KNaNiH}_{6}$ and $\mathrm{BaSrZnH}_{6}$ have VBM at $\mathrm{X}$, while that of $\mathrm{CaScMnH}_{6}$ and $\mathrm{AgZrMnH} 6$ is at $\mathrm{K}$ high-symmetric point in the reciprocal space. Thus, both CBM and VBM of $\mathrm{BaSrZnH}_{6}$ and $\mathrm{KNaNiH}_{6}$ reside at $\mathrm{X} \mathrm{K}$-point, indicating those materials are direct bandgap semiconductors. $\mathrm{BaNaRhH}_{6}, \mathrm{KNaNiH}_{6}, \mathrm{CaCsMnH}_{6}$, and $\mathrm{NaYRuH}_{6}$ materials have very flat bands near the Fermi level, which is indicated by zero energy. As shown by electronic band theory, the electron effective mass can be very high at the flat bands [55]. Our partial density of states (PDOS) studies reveal that d-orbitals of transition metal atoms reside at the $\mathrm{M}$ site dominate in the valence region near the Fermi level. Even though the transition metal atoms can be found at A and $\mathrm{A}^{\prime}$ sites, their pdos of d-orbitals are not significant near the Fermi level.

Table 7: The band gap, conduction band minimum (CBM), valence band maximum (VBM) and type of semiconductor for $\mathrm{AA}^{\prime} \mathrm{MH}_{6}$ materials.

\begin{tabular}{|c|c|c|c|c|}
\hline Material & Band Gap (eV) & CBM & VBM & Type \\
\hline $\mathrm{BaNaRhH}_{6}$ & 3.0181 & $\mathrm{X}$ & W & Indirect \\
\hline $\mathrm{BaSrZnH}_{6}$ & 1.5864 & $\mathrm{X}$ & $\mathrm{X}$ & Direct \\
\hline$\overline{\mathrm{BaCsAlH}_{6}}$ & 2.9382 & $\mathrm{G}$ & $\mathrm{X}$ & Indirect \\
\hline $\mathrm{SrTlIrH}_{6}$ & 1.6245 & $\mathrm{X}$ & $\mathrm{W}$ & Indirect \\
\hline $\mathrm{CsKSiH}_{6}$ & 2.1647 & G & $\mathrm{X}$ & Indirect \\
\hline $\mathrm{KNaNiH}_{6}$ & 3.3024 & $\mathrm{X}$ & $\mathrm{X}$ & Direct \\
\hline $\mathrm{NaYRuH}_{6}$ & 2.5322 & $\mathrm{X}$ & $\overline{\mathrm{W}}$ & Indirect \\
\hline $\mathrm{CaScMnH}_{6}$ & 1.2716 & $\mathrm{X}$ & $\mathrm{K}$ & Indirect \\
\hline $\mathrm{YZnMnH}_{6}$ & 1.6003 & $\mathrm{X}$ & $\mathrm{W}$ & Indirect \\
\hline $\mathrm{NaZrMnH}_{6}$ & 1.5694 & $\mathrm{X}$ & $\mathrm{K}$ & Indirect \\
\hline $\mathrm{AgZrMnH}_{6}$ & 1.4111 & $\mathrm{X}$ & $\mathrm{K}$ & Indirect \\
\hline $\mathrm{ScZnMnH}_{6}$ & 1.6732 & $\mathrm{X}$ & $\mathrm{W}$ & Indirect \\
\hline
\end{tabular}

\section{Conclusion}

In summary, we have performed a systematic study to find stable semiconductors from the cubic materials predicted by a generative adversarial network named CubicGAN. First, we analyzed all the ternary and quaternary materials to find 
Generative design of semiconductor materials
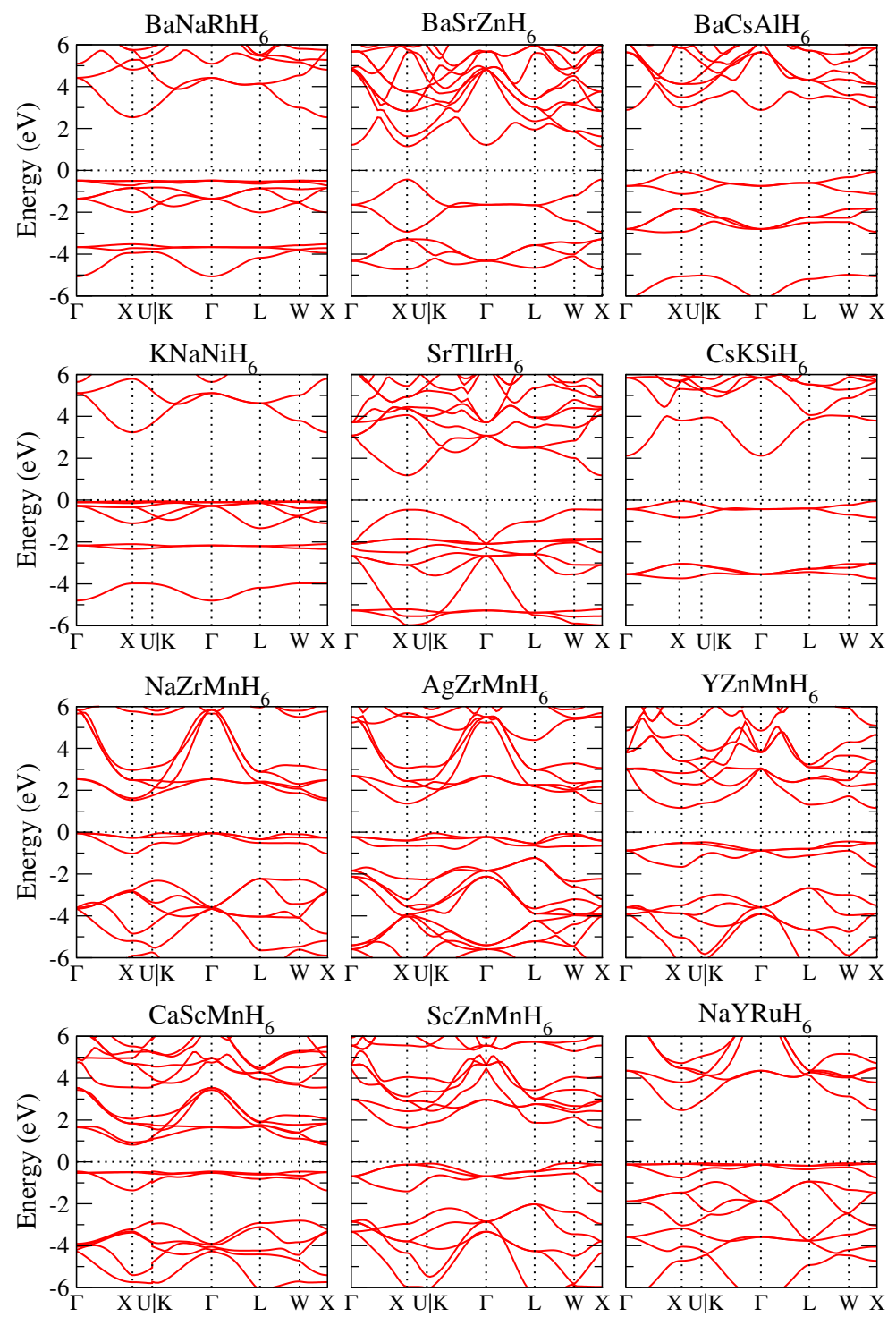

Figure 8: Band structures of $\mathrm{AA}^{\prime} \mathrm{MH}_{6}$ materials. Fermi energy marks zero energy.

which category has a higher probability of discovering a non-metal. A higher percentage $(\approx 56 \%)$ of ternary materials are metals, while most quaternary materials $(\approx 73 \%)$ are non-metals. And also, $\approx 80 \%$ of cubic ternary materials are metals, whereas $\approx 64 \%$ of cubic quaternary materials are non-metals. This indicates a higher probability of finding a cubic quaternary semiconductor. Next, we analyzed FI using an RFC model. Based on the features where FI is greater than $1 \%$, there is a low probability of finding a non-metal at higher values of Avg. availability of TM $(>0.4)$, the Avg. number of unfilled valence orbitals $(>0.76)$, and the Avg. number of unfilled valence d-orbitals $(>0.4)$. We applied our DNN classifier to the 323 mechanically and dynamically stable quaternary materials discovered by the CubicGAN model. DNN classified that 101 materials are semiconductors. Then, we carried out DFT calculations to investigate the thermodynamic stability and semiconductor properties of those 101 materials. It could be found that 12 materials with the chemical formulas in the form of $\mathrm{AA}^{\prime} \mathrm{MH}_{6}$ have non-zero bandgaps, zero energy-above-hull, and negative formation energies. Therefore, those are thermodynamically stable semiconductors. Next, we studied the structural, mechanical, and thermodynamic properties of those 12 stable semiconductors using DFT. Our studies show that $\mathrm{AA}^{\prime} \mathrm{MH}_{6}$ with $\mathrm{M}=\mathrm{Mn}$ and $\mathrm{NaYRuH}_{6}$ materials have different properties than the rest of the 12 materials. A-M and $\mathrm{A}-\mathrm{A}^{\prime}$ bond lengths of $\mathrm{AA}^{\prime} \mathrm{MnH}_{6}$ and $\mathrm{NaYRuH}_{6}$ materials are considerably lower than that of the other 6 materials. Moreover, A- $\mathrm{A}^{\prime}$ bonds are parallel to $\mathbf{a}, \mathbf{b}$ and $\mathbf{c}$ directions of the cubic unit cell. Therefore, Elevated $C_{i i}(i=1,2,3)$ elastic constants in $\mathrm{AA}^{\prime} \mathrm{MnH}_{6}$ and $\mathrm{NaYRuH}_{6}$ materials can be due to strong interactions between $\mathrm{A}$ and $\mathrm{A}^{\prime}$ atoms. $K, G$, and $Y$ modulus values are also considerably higher for former than later. Due to those mechanical property differences, $\mathrm{AA}^{\prime} \mathrm{MnH}_{6}$ and 
Generative design of semiconductor materials
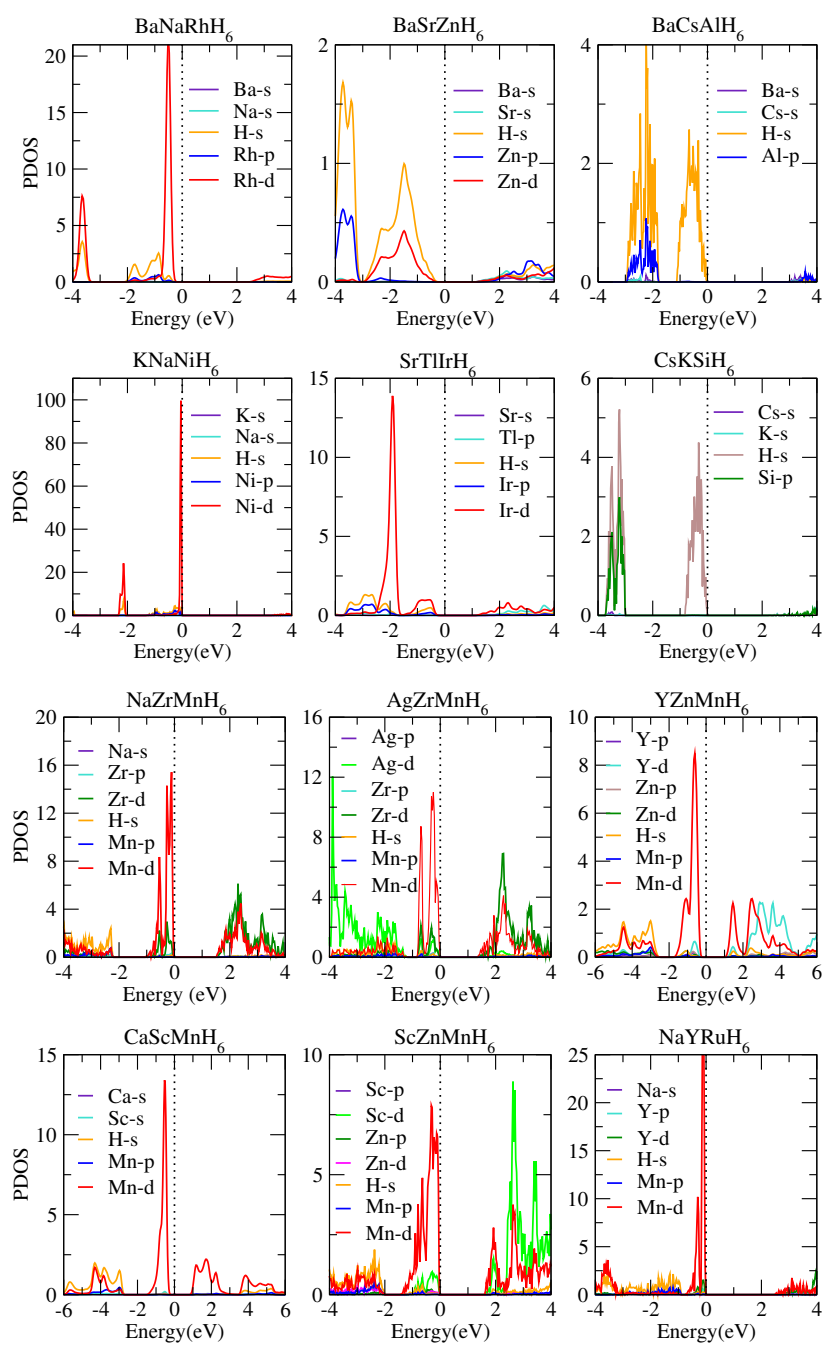

Figure 9: Partial density of states (PDOS) of $\mathrm{AA}^{\prime} \mathrm{MH}_{6}$ materials. Fermi energy marks zero energy.

$\mathrm{NaYRuH}_{6}$ materials exhibit smaller $C_{v}$ at low temperatures compared to the other 6 materials. Furthermore, our band structure calculations show that maximum bandgap $(\approx 3.3 \mathrm{eV})$ can be obtained from $\mathrm{KNaNiH}_{6}$, whereas minimum bandgap $(\approx 1.6 \mathrm{eV})$ is from $\mathrm{YZnMnH}_{6} . \mathrm{BaNaRhH}_{6}, \mathrm{BaCsAlH}_{6}, \mathrm{CsKSiH}_{6}, \mathrm{KNaNiH}_{6}$, and $\mathrm{NaYRuH}_{6}$ materials have bandgaps greater than $2 \mathrm{eV}$ implementing those are wide-bandgap semiconductors.

\section{Contribution}

Conceptualization, J.H. and E.S.; methodology, E.S.,Y.Z.; software, J.H.,Y.S.; resources, J.H., I.P.; writing-original draft preparation, E.S.,Y.Z.; writing-review and editing, J.H, I.P, and E.S.; visualization, E.S. and Y.Z.; supervision, J.H.; funding acquisition, J.H.

\section{Acknowledgement}

The research reported in this work was supported in part by National Science Foundation under the grant and 1940099 and 1905775. The views, perspectives, and content do not necessarily represent the official views of the NSF. We also would like to thank the support received from the department of computer science and engineering of the University of Moratuwa, Sri Lanka. 
Generative design of semiconductor materials

\section{References}

[1] Ricky W. Chuang, Rong-Xun Wu, Li-Wen Lai, and Ching-Ting Lee. Zno-on-gan heterojunction light-emitting diode grown by vapor cooling condensation technique. Applied Physics Letters, 91(23):231113, 2007.

[2] Lili Yu, Ahmad Zubair, Elton J. G. Santos, Xu Zhang, Yuxuan Lin, Yuhao Zhang, and Tomás Palacios. Highperformance wse 2 complementary metal oxide semiconductor technology and integrated circuits. Nano Letters, 15(8):4928-4934, 2015. PMID: 26192468.

[3] Martin A Green and Stephen P Bremner. Energy conversion approaches and materials for high-efficiency photovoltaics. Nature Materials, 16(1):23-34, January 2017.

[4] Yuxuan Lin, Xinming Li, Dan Xie, Tingting Feng, Yu Chen, Rui Song, He Tian, Tianling Ren, Minlin Zhong, Kunlin Wang, and Hongwei Zhu. Graphene/semiconductor heterojunction solar cells with modulated antireflection and graphene work function. Energy Environ. Sci., 6:108-115, 2013.

[5] Fumiyasu Oba and Yu Kumagai. Design and exploration of semiconductors from first principles: A review of recent advances. Applied Physics Express, 11(6):060101, may 2018.

[6] Samir Rom, Aishwaryo Ghosh, Anita Halder, and Tanusri Saha Dasgupta. Machine learning classification of binary semiconductor heterostructures. Phys. Rev. Materials, 5:043801, Apr 2021.

[7] H.K. Charles and G.K. Sujan. Microelectronic packaging: Electrical interconnections. In Reference Module in Materials Science and Materials Engineering. Elsevier, 2016.

[8] Md. Atikur Rahman. A review on semiconductors including applications and temperature effects in semiconductors. American Scientific Research Journal for Engineering, Technology, and Sciences, 7:50-70, 2014.

[9] Yoyo Hinuma, Taisuke Hatakeyama, Yu Kumagai, Lee A Burton, Hikaru Sato, Yoshinori Muraba, Soshi Iimura, Hidenori Hiramatsu, Isao Tanaka, Hideo Hosono, and Fumiyasu Oba. Discovery of earth-abundant nitride semiconductors by computational screening and high-pressure synthesis. Nature Communications, 7(1):11962, June 2016.

[10] J.B. Casady and R.W. Johnson. Status of silicon carbide (sic) as a wide-bandgap semiconductor for hightemperature applications: A review. Solid-State Electronics, 39(10):1409-1422, 1996.

[11] Carlos Ortiz, O.Eriksson, and Mattias Klintenberg. Data mining and accelerated electronic structure theory as a tool in the search for new functional materials. Computational Materials Science, 44:1042-1049, 2008.

[12] Wahyu Setyawan, Romain M. Gaume, Stephanie Lam, Robert S. Feigelson, and Stefano Curtarolo. Highthroughput combinatorial database of electronic band structures for inorganic scintillator materials. ACS Combinatorial Science, 13(4):382-390, 2011. PMID: 21644557.

[13] Shulin Luo, Tianshu Li, Xinjiang Wang, Muhammad Faizan, and Lijun Zhang. High-throughput computational materials screening and discovery of optoelectronic semiconductors. WIREs Computational Molecular Science, 11(1):e1489.

[14] Xin-Gang Zhao, Dongwen Yang, Yuanhui Sun, Tianshu Li, Lijun Zhang, Liping Yu, and Alex Zunger. Cu-in halide perovskite solar absorbers. Journal of the American Chemical Society, 139(19):6718-6725, 2017. PMID: 28430435.

[15] Yuheng Li and Kesong Yang. High-throughput computational design of organic-inorganic hybrid halide semiconductors beyond perovskites for optoelectronics. Energy Environ. Sci., 12:2233-2243, 2019.

[16] Ian Goodfellow, Jean Pouget-Abadie, Mehdi Mirza, Bing Xu, David Warde-Farley, Sherjil Ozair, Aaron Courville, and Yoshua Bengio. Generative adversarial nets. Advances in neural information processing systems, $27,2014$.

[17] Martin Arjovsky, Soumith Chintala, and Léon Bottou. Wasserstein generative adversarial networks. In International conference on machine learning, pages 214-223. PMLR, 2017.

[18] Ishaan Gulrajani, Faruk Ahmed, Martin Arjovsky, Vincent Dumoulin, and Aaron Courville. Improved training of wasserstein gans. arXiv preprint arXiv:1704.00028, 2017.

[19] Asma Nouira, Nataliya Sokolovska, and Jean-Claude Crivello. Crystalgan: learning to discover crystallographic structures with generative adversarial networks. arXiv preprint arXiv:1810.11203, 2018.

[20] Jun-Yan Zhu, Taesung Park, Phillip Isola, and Alexei A Efros. Unpaired image-to-image translation using cycle-consistent adversarial networks. In Proceedings of the IEEE international conference on computer vision, pages 2223-2232, 2017.

[21] Sungwon Kim, Juhwan Noh, Geun Ho Gu, Alan Aspuru-Guzik, and Yousung Jung. Generative adversarial networks for crystal structure prediction. ACS central science, 6(8):1412-1420, 2020. 
Generative design of semiconductor materials

[22] Yong Zhao, Mohammed Al-Fahdi, Ming Hu, Edirisuriya Siriwardane, Yuqi Song, Alireza Nasiri, and Jianjun Hu. High-throughput discovery of novel cubic crystal materials using deep generative neural networks. arXiv preprint arXiv:2102.01880, 2021.

[23] James E Saal, Scott Kirklin, Muratahan Aykol, Bryce Meredig, and Christopher Wolverton. Materials design and discovery with high-throughput density functional theory: the open quantum materials database (oqmd). Jom, 65(11):1501-1509, 2013.

[24] Scott Kirklin, James E Saal, Bryce Meredig, Alex Thompson, Jeff W Doak, Muratahan Aykol, Stephan Rühl, and Chris Wolverton. The open quantum materials database (oqmd): assessing the accuracy of dft formation energies. npj Computational Materials, 1(1):1-15, 2015.

[25] Anubhav Jain, Shyue Ping Ong, Geoffroy Hautier, Wei Chen, William Davidson Richards, Stephen Dacek, Shreyas Cholia, Dan Gunter, David Skinner, Gerbrand Ceder, et al. Commentary: The materials project: A materials genome approach to accelerating materials innovation. Apl Materials, 1(1):011002, 2013.

[26] G Bergerhoff, ID Brown, F Allen, et al. Crystallographic databases. International Union of Crystallography, Chester, 360:77-95, 1987.

[27] Tian Xie and Jeffrey C Grossman. Crystal graph convolutional neural networks for an accurate and interpretable prediction of material properties. Physical review letters, 120(14):145301, 2018.

[28] Anubhav Jain, Shyue Ping Ong, Geoffroy Hautier, Wei Chen, William Davidson Richards, Stephen Dacek, Shreyas Cholia, Dan Gunter, David Skinner, Gerbrand Ceder, and Kristin a. Persson. The Materials Project: A materials genome approach to accelerating materials innovation. APL Materials, 1(1):011002, 2013.

[29] Shyue Ping Ong, William Davidson Richards, Anubhav Jain, Geoffroy Hautier, Michael Kocher, Shreyas Cholia, Dan Gunter, Vincent L. Chevrier, Kristin A. Persson, and Gerbrand Ceder. Python Materials Genomics (pymatgen): A robust, open-source python library for materials analysis. Computational Materials Science, 68:314-319, February 2013.

[30] François Chollet et al. Keras. https://keras.io, 2015.

[31] Martín Abadi, Ashish Agarwal, Paul Barham, Eugene Brevdo, Zhifeng Chen, Craig Citro, Greg S. Corrado, Andy Davis, Jeffrey Dean, Matthieu Devin, Sanjay Ghemawat, Ian Goodfellow, Andrew Harp, Geoffrey Irving, Michael Isard, Yangqing Jia, Rafal Jozefowicz, Lukasz Kaiser, Manjunath Kudlur, Josh Levenberg, Dandelion Mané, Rajat Monga, Sherry Moore, Derek Murray, Chris Olah, Mike Schuster, Jonathon Shlens, Benoit Steiner, Ilya Sutskever, Kunal Talwar, Paul Tucker, Vincent Vanhoucke, Vijay Vasudevan, Fernanda Viégas, Oriol Vinyals, Pete Warden, Martin Wattenberg, Martin Wicke, Yuan Yu, and Xiaoqiang Zheng. TensorFlow: Large-scale machine learning on heterogeneous systems, 2015. Software available from tensorflow.org.

[32] F. Pedregosa, G. Varoquaux, A. Gramfort, V. Michel, B. Thirion, O. Grisel, M. Blondel, P. Prettenhofer, R. Weiss, V. Dubourg, J. Vanderplas, A. Passos, D. Cournapeau, M. Brucher, M. Perrot, and E. Duchesnay. Scikit-learn: Machine learning in Python. Journal of Machine Learning Research, 12:2825-2830, 2011.

[33] G. Kresse and J. Hafner. ab initio. Phys. Rev. B, 47:558-561, Jan 1993.

[34] G. Kresse and J. Hafner. ab initio. Phys. Rev. B, 49:14251-14269, May 1994.

[35] J. Furthmüller G. Kresse. Efficiency of ab initio total energy calculations for metals and semiconductors using a plane-wave basis set. Comput. Mater. Sci., 6:15-50, jul 1996.

[36] G. Kresse and J. Furthmüller. Efficient iterative schemes for ab initio total-energy calculations using a plane-wave basis set. Phys. Rev. B, 54:11169-11186, Oct 1996.

[37] P. E. Blöchl. Projector augmented-wave method. Phys. Rev. B, 50:17953-17979, Dec 1994.

[38] G. Kresse and D. Joubert. From ultrasoft pseudopotentials to the projector augmented-wave method. Phys. Rev. B, 59:1758-1775, Jan 1999.

[39] John P. Perdew, Kieron Burke, and Matthias Ernzerhof. Generalized gradient approximation made simple. Phys. Rev. Lett., 77:3865-3868, Oct 1996.

[40] John P. Perdew, Kieron Burke, and Matthias Ernzerhof. Generalized gradient approximation made simple [phys. rev. lett. 77, 3865 (1996)]. Phys. Rev. Lett., 78:1396-1396, Feb 1997.

[41] A Togo and I Tanaka. First principles phonon calculations in materials science. Scr. Mater., 108:1-5, Nov 2015.

[42] Stefano Baroni, Stefano de Gironcoli, Andrea Dal Corso, and Paolo Giannozzi. Phonons and related crystal properties from density-functional perturbation theory. Rev. Mod. Phys., 73:515-562, Jul 2001. 
Generative design of semiconductor materials

[43] Vei Wang, Nan Xu, Jin-Cheng Liu, Gang Tang, and Wen-Tong Geng. Vaspkit: A user-friendly interface facilitating high-throughput computing and analysis using vasp code. Computer Physics Communications, 267:108033, Oct 2021.

[44] R Hill. The elastic behaviour of a crystalline aggregate. Proceedings of the Physical Society. Section A, 65(5):349354, may 1952.

[45] K. Kadir, D. Moser, M. Münzel, and D. Noréus. Investigation of counterion influence on an octahedral irh6complex in the solid state hydrides aaeirh6 $(\mathrm{a}=\mathrm{na}, \mathrm{k}$ and $\mathrm{ae}=\mathrm{ca}, \mathrm{sr}, \mathrm{ba}$, and eu) with a new structure type. Inorganic Chemistry, 50(23):11890-11895, 2011. PMID: 22035072.

[46] Denis Zolotariov and Ph.D., Kharkov, Ukraine, https://orcid.org/0000-0003-4907-7810. Development of the approximating functions method for problems in a planar waveguide with constant polarization. Int. j. math. comput. res., 09(12), December 2021.

[47] Website Https //Www Shimbaleva Com and Inna Shimbaleva. Hidden treasures of music. Independently Published, October 2021.

[48] The Materials Project. Materials data on nacah6ir by materials project. 52020.

[49] Félix Mouhat and François-Xavier Coudert. Necessary and sufficient elastic stability conditions in various crystal systems. Physical review B, 90(22):224104, 2014.

[50] Richard Hill. The elastic behaviour of a crystalline aggregate. Proceedings of the Physical Society. Section A, 65(5):349, 1952.

[51] C. Li and Z. Wang. 9 - computational modelling and ab initio calculations in max phases - i. In I.M. Low, editor, Advances in Science and Technology of Mn+1AXn Phases, pages 197-222. Woodhead Publishing, 2012.

[52] Hannes Raebiger, Stephan Lany, and Alex Zunger. Charge self-regulation upon changing the oxidation state of transition metals in insulators. Nature, 453(7196):763-766, June 2008.

[53] Kiyoshi Takahashi, Akihiko Yoshikawa, and Adarsh Sandhu. Wide bandgap semiconductors. Springer-Verlag Berlin Heidelberg., page 239, 2007.

[54] Jose Millan, Philippe Godignon, Xavier Perpiñà, Amador Pérez-Tomás, and José Rebollo. A survey of wide bandgap power semiconductor devices. IEEE transactions on Power Electronics, 29(5):2155-2163, 2013.

[55] Chengyong Zhong, Yuee Xie, Yuanping Chen, and Shengbai Zhang. Coexistence of flat bands and dirac bands in a carbon-kagome-lattice family. Carbon, 99:65-70, 2016. 